\title{
Parent-Child Feedback Predicts Sibling Contrast: Using Twin Studies to Test Theories of Parent-Offspring Interaction in Infant Behavior
}

\author{
Lindon J. Eaves and Judy L. Silberg \\ Virginia Institute for Psychiatric and Behavioral Genetics, Virginia Commonwealth University, Richmond,Virginia, United States of America
}

S everal studies report apparent sibling contrast effects in analyses of twin resemblance. In the presence of genetic differences, contrast effects reduce the dizygotic (DZ) twin correlation relative to that in monozygotic (MZ) twins and produce higher DZ than MZ variance. Explanations of contrast effects are typically cast in terms of direct social interaction between twins or an artifact of the process of rating children by their parents. We outline a model for sibling imitation and contrast effects that depends on social interaction between parents and children. In addition to predicting the observed pattern of twin variances and covariances, the parental mediation of child imitation and contrast effects leads to differences in the variance of parents of $M Z$ and $D Z$ twins and differences between the correlations of parents with their $M Z$ and DZ children.

It has long been recognized that reciprocal social interaction between siblings affects the pattern of variances and correlations in monozygotic (MZ) and dizygotic (DZ) twins (Carey, 1986; Eaves, 1976). Sibling competition and contrast effects lead to a characteristic reduction of the correlation in $\mathrm{DZ}$ twins relative to one half of the $M Z$ correlation, and a $M Z$ variance less than that of $\mathrm{DZ}$ twins. In extreme cases, the $\mathrm{DZ}$ correlation may be negative, which can never be anticipated under the conventional model for genetic and environmental influences.

Several studies have replicated the apparent effect of sibling contrast on infant temperament, especially difficult temperament (Goldsmith et al., 1997; Saudino, 2003). Given that it is difficult to envision the direct interaction of very young twins, it has often been assumed that the contrast effects are an artifact of an implicit tendency for mothers to contrast their children with one another when rating behavior. However, it remains difficult to understand why some traits, such as difficult temperament, consistently show rater contrast effects while others do not. A similar difficulty arises in explaining the consistent finding of sibling contrast effects in twin studies of childhood hyperactivity (Simonoff et al., 1998).

We present a model in which interaction between parent and child not only explains the widespread finding of contrast effects but makes additional quantitative predictions that may be tested empirically. Such a model has greater theoretical power than current explanations.

\section{Model}

Difficult temperament is often conceived as a primary index of underlying inherited neurobiological processes, hence it is expected that differences in temperament should reflect principally genetic effects and any random environmental effects that influence individuals. Instead we propose that 'difficult temperament' even in infants is the product of an already long dynamic developmental history of feedback between the behavior of children and the calibrated response of their parents. We argue that difficult behavior of the inherently reactive child evokes behavior from the parent that ameliorates child behavior through negative feedback. What appears in conventional twin studies as sibling contrast effects should be viewed not as a direct negative reciprocal interaction between twins but as a consequence of the pathway, mediated through parental behavior in response to difficult behavior in the child. Since the path from a child's behavior to parental response is positive and the reciprocal path from parental response to child behavior is negative, we anticipate a net negative pathway, mediated through parent behavior, from one twin to the other. This, in turn, is expected to result in apparent sibling contrast effects in twin data.

The model is presented more formally in Figure 1.

Received 21 October, 2004; accepted 28 November, 2004

Address for correspondence: Professor Lindon Eaves, Virginia Institute for Psychiatric and Behavioral Genetics, Virginia Commonwealth University, Richmond,VA, 23298,USA.E-mail: eaves@mail2.vcu.edu 


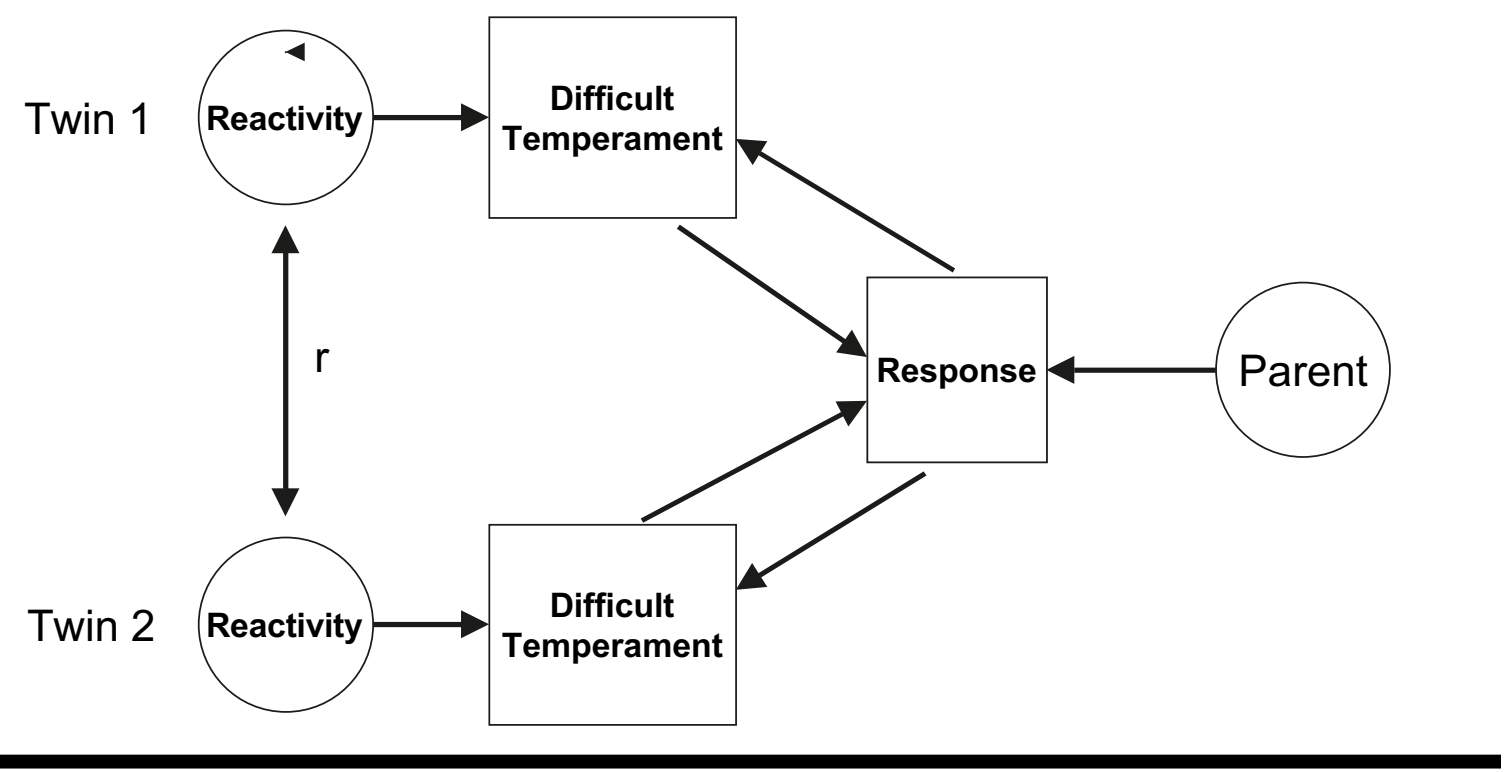

Figure 1

Model for reciprocal interaction between infant temperament and parental behavior.

If we write $\mathrm{R}$ for the $3 \times 3$ matrix of standardized covariances between reactivity in first and second twins and the responsiveness of parents, and B for the matrix of pathways between the infant phenotypes for difficult temperament and the behavioral response of the parent, the resultant covariance matrix between child temperament and parental response at dynamic equilibrium is:

$$
\mathrm{C}=(\mathrm{I}-\mathrm{B})^{-1} \mathrm{R}(\mathrm{I}-\mathrm{B})^{-1}
$$

In the simple case where infant reactivity is correlated between twins and independent of parental responsiveness we have:

$$
\mathrm{R}=\quad \begin{array}{lll}
1 & r & 0 \\
r & 1 & 0 \\
0 & 0 & 1
\end{array}
$$

$r$ being the correlation between reactivity of first and second twins. We expect that $r$ will differ for MZ and DZ twins as a function of genetic differences.

The $j$ th element of the $i$ th row of B is the pathway from the $j$ th observed variable to the $i$ th outcome. $\mathrm{B}$ will be null except for the elements reflecting the interaction between child temperament and parental response. That is, in our case:

$$
\mathrm{B}=\quad \begin{array}{lll}
0 & 0 & q \\
0 & 0 & q \\
p & p & 0
\end{array}
$$

Equation [1] may be evaluated numerically for given $\mathrm{MZ}$ and DZ correlations in reactivity and various positive and negative values of the reciprocal paths, $p$ and $q$, between infant temperament and parental behavior. We consider cases $|p|=|q|=0.2$ and 0.4 , with combinations of positive and negative values of $p$ and $q$. We assume the MZ and DZ correlations for reactivity are .5 and .25 respectively, corresponding to fairly typical twin correlations for measures of behavior that show intermediate narrow heritability.

\section{Results}

Expected phenotypic variances and correlations are given in Table 1 for MZ and DZ twins together with the variances of parental response and the correlation between infant temperament and parent response for both types of twin family.

The pattern of twin variances and correlations follow those expected in the presence of sibling interactions (Carey, 1986; Eaves, 1976). When the signs of $p$ and $q$ are the same, there is a net positive pathway from the behavior of one twin to the other, and the statistics follow what is to be expected in the presence of sibling cooperation or imitation effects. That is, the correlation for DZ twins is increased relative to that for $M Z$ pairs in a manner superficially consistent with the effects of the shared environment. This also results in the phenotypic variance for MZs exceeding that for DZs. When $p$ and $q$ have opposite signs (e.g., when a child's temperament evokes a positive reaction from the parent that then exercises a negative feedback on child behavior) we find the expected net effect of sibling contrast. That is, the correlation for $\mathrm{DZ}$ pairs is reduced relative to that 
Table 1.

Expected Variances $(\boldsymbol{V}$ and Correlations $(r)$ for Twin and Parental Behavior in the Presence of Reciprocal Interaction Between Parent and Child

\begin{tabular}{|c|c|c|c|c|c|c|c|c|c|}
\hline \multicolumn{2}{|c|}{ Interaction } & \multicolumn{4}{|c|}{ MZ pairs } & \multicolumn{4}{|c|}{ DZ pairs } \\
\hline$p$ & $q$ & $V_{\text {twin }}$ & $r_{\text {twin }}$ & $V_{\text {parent }}$ & $r_{p / o}$ & $V_{\text {twin }}$ & $r_{\text {twin }}$ & $V_{\text {parent }}$ & $r_{p / 0}$ \\
\hline .2 & .2 & 1.18 & .58 & 1.32 & .47 & 1.16 & .35 & 1.30 & .43 \\
\hline .4 & .4 & 2.22 & .77 & 3.20 & .81 & 2.07 & .64 & 3.03 & .77 \\
\hline-.2 & -.2 & 1.18 & .58 & 1.32 & -.47 & 1.16 & .35 & 1.30 & -.43 \\
\hline-.4 & -.4 & 2.22 & .77 & 3.20 & -.81 & 2.07 & .64 & 3.03 & -.77 \\
\hline .2 & -.2 & 0.93 & .46 & 0.96 & .09 & 0.95 & .21 & 0.94 & .05 \\
\hline .4 & -.4 & 0.77 & .35 & 0.85 & .14 & 0.83 & .09 & 0.80 & .07 \\
\hline-.2 & .2 & 0.93 & .43 & 0.96 & -.09 & 0.95 & .21 & 0.94 & -.05 \\
\hline-.4 & .4 & 0.77 & .35 & 0.85 & -.14 & 0.83 & .09 & 0.80 & -.07 \\
\hline
\end{tabular}

for MZs and to a smaller total variance in $\mathrm{MZ}$ compared with DZ twins.

A striking feature of the results in the table is the predicted pattern of parental variances and parent-offspring correlations. The variances in the behavior of parents of $\mathrm{MZ}$ twins are consistently greater than those of $\mathrm{DZ}$ twins regardless of the relative signs of $p$ and $q$. This implies the influence of children on their parents or evocative genotype environment correlation (rGE; Plomin et al., 1983).

The impact of the parent-child interaction on the correlations between parental behavior and child temperament is also of particular significance. If children did not influence the behavior of their parents, we expect the parent-offspring correlations to be the same for $M Z$ and $D Z$ twins. However, the impact of twins on their parents results in the parents and their $M Z$ twin children showing greater absolute correlations than parents and their DZ twin children, another consequence of evocative rGE. As might be expected, parent-offspring correlations are higher in absolute value when $p$ and $q$ have the same sign. When the child-parent path, $p$, is negative, so are the parent offspring correlations.

\section{$\overline{\text { Discussion }}$}

The model we have considered provides a theoretical framework for examining the patterns of parent-offspring interaction for infant behavior that can explain effects such as apparent sibling contrast without recourse either to a direct interaction between twins or to an artifact of comparative rating of children by their parents. Furthermore, the model predicts patterns of correlation between parent and child behavior consistent with evocative g-e correlation in which the behavior of parents is a response to the behavior of the child. Our illustrative model may be extended in several ways without affecting the basic concept. Thus, although we have focused on 'twinparent' families, the same model can be extended to other family sizes and configurations such as parents and singletons or multiple sibings (Carey, 1986; Eaves, 1976). We also assume that child temperament and parental treatment have no extraneous correlation due not explained by parent-child interaction. If this is not the case, the $\mathrm{R}$ matrix may be modified accordingly, at least in theory. The lack of reciprocal twin interaction in singletons is expected to reduce the child variance in singletons relative to that in twins. Although we have not conducted a formal power analysis, we anticipate that the power for tests of interaction is greater for competition/contrast effects than for those of imitation since the latter tend to resemble the pattern of increased (positive) correlation between relatives due to shared environmental effects. Making allowance for the parental mediation of sibling interaction may provide a theoretical framework to explain anomalies in patterns of variance and correlation in studies of young twins and their parents that have hitherto been dismissed for want of a coherent explanation.

\section{Acknowledgments}

This work was supported by the Bank of America Carman Trust Award (JS) and the Clinical Research Center of the University of Puerto Rico, Medical Sciences Campus (1P20RR11126). We thank Greg Carey for his helpful review of an early version of this paper.

\section{References}

Carey, G. (1986). Sibling imitation and contrast effects. Behavior Genetics, 16, 319-341.

Eaves, L. J. (1976). A model for sibling effects in man. Heredity, 36, 205-214.

Goldsmith, H., Buss., K. A., \& Lemery, K. S. (1997). Toddler and childhood temperament: Expanded content, stronger genetic evidence, new evidence for the importance of the environment. Developmental Psychology, 33, 891-905. 
Plomin, R., DeFries, J. C., \& Loehlin, J. C. (1977). Genotype-environment interaction and correlation in the analysis of human behavior. Psychological Bulletin, 84, 309-322.

Saudino, K. J. (2003). Parent ratings of infant temperament: Lessons from twin studies. Infant Behavior and Development, 26, 100-107.
Scarr, S., \& McCartney, K. (1983). How people make their own environments: A theory of genotype-environment effects. Child Development, 54, 424-435.

Simonoff, E., Pickles, A., Hervas, A., Silberg, J. L., Rutter, M., \& Eaves, L. J. (1998). Genetic influences on childhood hyperactivity: Contrast effects imply parental rating bias, not sibling interaction. Psychological Medicine, 28, 825-837. 